\title{
Dynamics of a class of neutral three neurons network with delay
}

\author{
Ming Liu, Chunrui Zhang ${ }^{*}$ and Xiaofeng Xu
}

\section{"Correspondence:}

math@nefu.edu.cn

Department of mathematics,

Northeast Forestry University,

Harbin, 150040, P.R. China

\begin{abstract}
In this paper, a class of neutral neural networks with delays is investigated. The linear stability of the model is studied. It is found that a Hopf bifurcation also occurs when some delays pass through a sequence of critical values. The direction of the Hopf bifurcations and the stability of bifurcating periodic solutions are determined by using the normal form method and center manifold theory. The existence of a permanent oscillation is established using Chafee's criterion. Numerical simulations are performed to support the analytical results.
\end{abstract}

Keywords: neutral neural network; stability; Hopf bifurcation; permanent oscillation

\section{Introduction}

Since 1990s, the theories and applications of neural networks with delays have been greatly developed. It is well known that many important mathematical models from physics, biology, etc. can be written in neurons network models. In 2008, Li and Yuan considered a Hopfield-type network of three identical neurons coupled in any possible way in [1]:

$$
\begin{aligned}
& \dot{x}_{1}(t)=-x_{1}(t)+a f\left(x_{1}\left(t-\tau_{s}\right)\right)+a_{12} b g\left(x_{2}\left(t-\tau_{n}\right)\right)+a_{13} b g\left(x_{3}\left(t-\tau_{n}\right)\right), \\
& \dot{x}_{2}(t)=-x_{2}(t)+a_{21} b g\left(x_{1}\left(t-\tau_{n}\right)\right)+a f\left(x_{2}\left(t-\tau_{s}\right)\right)+a_{23} b g\left(x_{3}\left(t-\tau_{n}\right)\right), \\
& \dot{x}_{3}(t)=-x_{3}(t)+a_{31} b g\left(x_{1}\left(t-\tau_{n}\right)\right)+a_{32} b g\left(x_{2}\left(t-\tau_{n}\right)\right)+a f\left(x_{3}\left(t-\tau_{s}\right)\right) .
\end{aligned}
$$

Due to the finite speed of the switching and transmission of signals, neutral behavior does exist in the neural network with delays and should be incorporated. For this reason, we improve the original model in which the neutral behavior was added and obtain the following forms [2]:

$$
\begin{aligned}
\dot{x}_{1}(t)= & -x_{1}(t)+a f\left(x_{1}\left(t-\tau_{s}\right)\right)+a_{12} b g\left(x_{2}\left(t-\tau_{n}\right)\right)+a_{13} b g\left(x_{3}\left(t-\tau_{n}\right)\right) \\
& +a_{2} f_{2}\left(\dot{x}_{1}\left(t-\tau_{s}\right)\right)+b_{12} b_{2} g_{2}\left(\dot{x}_{2}\left(t-\tau_{n}\right)\right)+b_{13} b_{2} g_{2}\left(\dot{x}_{3}\left(t-\tau_{n}\right)\right), \\
\dot{x}_{2}(t)= & -x_{2}(t)+a_{21} b g\left(x_{1}\left(t-\tau_{n}\right)\right)+a f\left(x_{2}\left(t-\tau_{s}\right)\right)+a_{23} b g\left(x_{3}\left(t-\tau_{n}\right)\right) \\
& +b_{21} b_{2} g_{2}\left(\dot{x}_{1}\left(t-\tau_{n}\right)\right)+a_{2} f_{2}\left(\dot{x}_{2}\left(t-\tau_{s}\right)\right)+b_{23} b_{2} g_{2}\left(\dot{x}_{3}\left(t-\tau_{n}\right)\right), \\
\dot{x}_{3}(t)= & -x_{3}(t)+a_{31} b g\left(x_{1}\left(t-\tau_{n}\right)\right)+a_{32} b g\left(x_{2}\left(t-\tau_{n}\right)\right)+a f\left(x_{3}\left(t-\tau_{s}\right)\right) \\
& +b_{31} b_{2} g_{2}\left(\dot{x}_{1}\left(t-\tau_{n}\right)\right)+b_{32} b_{2} g_{2}\left(\dot{x}_{2}\left(t-\tau_{n}\right)\right)+a_{2} f_{2}\left(\dot{x}_{3}\left(t-\tau_{s}\right)\right),
\end{aligned}
$$

@2013 Liu et al.; licensee Springer. This is an Open Access article distributed under the terms of the Creative Commons Attribution License (http://creativecommons.org/licenses/by/2.0), which permits unrestricted use, distribution, and reproduction in any medium, provided the original work is properly cited. 
where $a_{i j}, b_{i j}(i \neq j, i, j=1,2,3)$ have the values 1 or 0 , depending whether the cells from $j$ to $i$ are connected or not; $a, b, a_{2}, b_{2} \in R$ denote the strength in self-connection and neighboring-connection, respectively; $\tau_{s}, \tau_{n} \geq 0$ are the corresponding time delays. Furthermore, $f, g, f_{2}, g_{2}$ are assumed to be adequately smooth and to satisfy the following conditions:

$$
\begin{aligned}
& \left(\mathrm{H}_{1}\right) \quad f(0)=g(0)=f_{2}(0)=g_{2}(0)=0, \\
& \left(\mathrm{H}_{2}\right) \quad f^{\prime}(0)=g^{\prime}(0)=f_{2}^{\prime}(0)=g_{2}^{\prime}(0)=1 .
\end{aligned}
$$

Then we derive the stability of this system and conditions of existence of the bifurcation with $a_{12}=a_{13}=a_{23}=a_{32}=1, a_{21}=a_{31}=0 ; b_{12}=b_{13}=b_{23}=b_{32}=1, b_{21}=b_{31}=0$, $\tau_{s}=\tau_{n}=\tau$. The remainder of this paper is organized as follows. In Section 2 we introduce the stability of the equilibrium point and the conditions of existence of a local Hopf bifurcation. We are devoted to establishing the direction and stability of the Hopf bifurcation in Section 3. In Section 4 we discuss the existence of a permanent oscillation. Finally, we carry out some numerical simulation to support the analysis result in Section 5.

\section{Stability and Hopf bifurcation analysis}

In this section, we let $a_{12}=a_{13}=a_{23}=a_{32}=1, a_{21}=a_{31}=0 ; b_{12}=b_{13}=b_{23}=b_{32}=1, b_{21}=$ $b_{31}=0$, then we have

$$
\begin{aligned}
\dot{x}_{1}(t)= & -x_{1}(t)+a f\left(x_{1}(t-\tau)\right)+b g\left(x_{2}(t-\tau)\right)+b g\left(x_{3}(t-\tau)\right) \\
& +a_{2} f_{2}\left(\dot{x}_{1}(t-\tau)\right)+b_{2} g_{2}\left(\dot{x}_{2}(t-\tau)\right)+b_{2} g_{2}\left(\dot{x}_{3}(t-\tau)\right), \\
\dot{x}_{2}(t)= & -x_{2}(t)+a f\left(x_{2}(t-\tau)\right)+b g\left(x_{3}(t-\tau)\right) \\
& +a_{2} f_{2}\left(\dot{x}_{2}(t-\tau)\right)+b_{2} g_{2}\left(\dot{x}_{3}(t-\tau)\right), \\
\dot{x}_{3}(t)= & -x_{3}(t)+b g\left(x_{2}(t-\tau)\right)+a f\left(x_{3}(t-\tau)\right) \\
& +b_{2} g_{2}\left(\dot{x}_{2}(t-\tau)\right)+a_{2} f_{2}\left(\dot{x}_{3}(t-\tau)\right) .
\end{aligned}
$$

Under the given hypotheses $\left(\mathrm{H}_{1}\right)$ and $\left(\mathrm{H}_{2}\right)$, it is easy to check that $x=(0,0,0)^{T}$ is an equilibrium point of system (2.1). By using a similar method to that in [3], we have the following results on stability to system (2.1).

Theorem 1 Let $\left|a_{2}+b_{2}\right|<1$ and $\left|a_{2}-b_{2}\right|<1$.

(1) If $(a, b) \in D_{1}$, then the zero solution of system (2.1) is absolutely stable.

(2) If $(a, b) \in D_{2}$, then the zero solution of system (2.1) is conditionally stable, i.e., $\tau \in\left[0, \tau_{0}\right)$, the zero solution of system (2.1) is asymptotically stable; $\tau>\tau_{0}$, the zero solution of system (2.1) is unstable,

(a) if $a<-1$, system (2.1) undergoes a Hopf bifurcation at the origin when $\tau=\tau_{1 j}$, $j=0,1,2, \ldots$,

(b) if $a+b<-1$, system (2.1) undergoes a Hopf bifurcation at the origin when $\tau=\tau_{2 j}$, $j=0,1,2, \ldots$,

(c) if $a-b<-1$, system (2.1) undergoes a Hopf bifurcation at the origin when $\tau=\tau_{3 j}$, $j=0,1,2, \ldots$ 


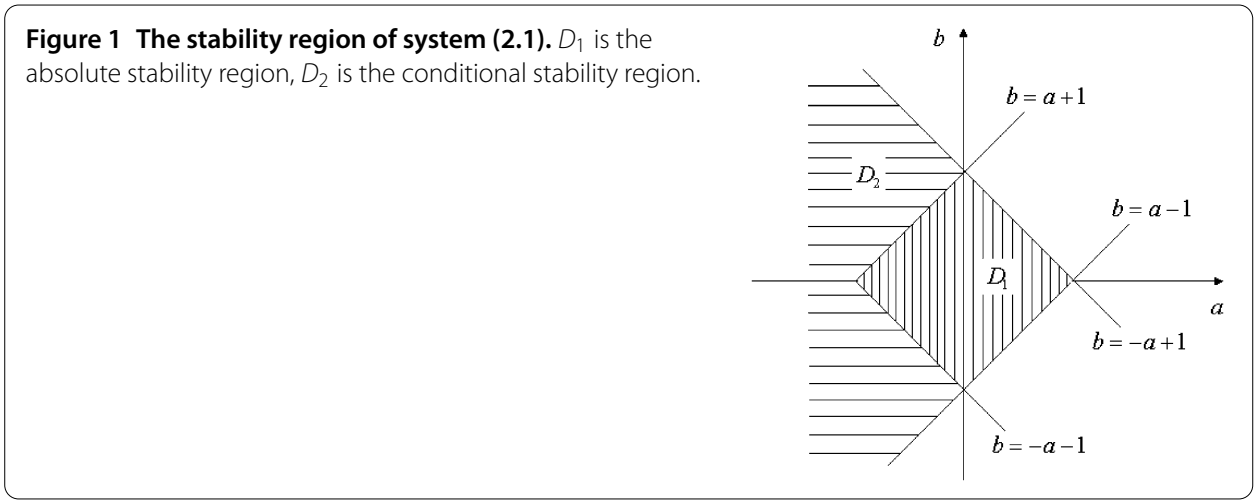

with

$$
\begin{aligned}
\tau_{1 j} & =\sqrt{\frac{1-a_{2}^{2}}{a^{2}-1}}\left[\arccos \frac{a a_{2}+1}{a+a_{2}}+2 \pi j\right], \\
\tau_{2 j} & =\sqrt{\frac{1-\left(a_{2}+b_{2}\right)^{2}}{(a+b)^{2}-1}}\left[\arccos \frac{(a+b)\left(a_{2}+b_{2}\right)+1}{a+b+a_{2}+b_{2}}+2 \pi j\right], \\
\tau_{3 j} & =\sqrt{\frac{1-\left(a_{2}-b_{2}\right)^{2}}{(a-b)^{2}-1}}\left[\arccos \frac{(a-b)\left(a_{2}-b_{2}\right)+1}{a-b+a_{2}-b_{2}}+2 \pi j\right], \\
\tau_{0} & =\min \left[\tau_{10}, \tau_{20}, \tau_{30}\right],
\end{aligned}
$$

where $D_{1}$ and $D_{2}$ are shown in Figure 1.

Proof From hypotheses $\left(\mathrm{H}_{1}\right)$ and $\left(\mathrm{H}_{2}\right)$, the characteristic equation associated with the linearization of system (2.1) is

$$
\Delta_{1}(\lambda) \Delta_{2}(\lambda) \Delta_{3}(\lambda)=0
$$

where

$$
\begin{aligned}
& \Delta_{1}(\lambda)=\lambda+1-\left(a+a_{2} \lambda\right) e^{-\lambda \tau}, \\
& \Delta_{2}(\lambda)=\lambda+1-\left(a+b+\left(a_{2}+b_{2}\right) \lambda\right) e^{-\lambda \tau}, \\
& \Delta_{3}(\lambda)=\lambda+1-\left(a-b+\left(a_{2}-b_{2}\right) \lambda\right) e^{-\lambda \tau} .
\end{aligned}
$$

Separately analyzing the roots of $\Delta_{i}(\lambda)=0(i=1,2,3)$, by using the method in [3], we have the following results.

If $-1 \leq a<1$, then all roots of $\Delta_{1}(\lambda)=0$ have negative real parts for all $\tau \geq 0$. If $a<-1$, then $\Delta_{1}(\lambda)=0$ has a pair of purely imaginary roots when $\tau=\tau_{1 j}$.

If $-1 \leq a+b<1$, then all roots of $\Delta_{2}(\lambda)=0$ have negative real parts for all $\tau \geq 0$. If $a+b<-1$, then $\Delta_{2}(\lambda)=0$ has a pair of purely imaginary roots when $\tau=\tau_{2 j}$.

If $-1 \leq a-b<1$, then all roots of $\Delta_{3}(\lambda)=0$ have negative real parts for all $\tau \geq 0$. If $a-b<-1$, then $\Delta_{3}(\lambda)=0$ has a pair of purely imaginary roots when $\tau=\tau_{3 j}$.

Additionally, all roots of $\Delta_{i}(\lambda)=0(i=1,2,3)$ have negative real parts when $\tau=0$ and $\left.\operatorname{Re} \frac{d \lambda}{d \tau}\right|_{\tau=\tau_{i j}}>0(i=1,2,3)$ is satisfied. 
Summarizing the conclusions above, the proof is completed.

\section{Properties of Hopf bifurcation}

In the previous section, we have obtained the sufficient conditions for system (2.1) to undergo a Hopf bifurcation at the origin with $\tau$ as a bifurcation parameter. In this section, we shall investigate the direction of the Hopf bifurcation and stability of bifurcating periodic solutions by taking $f_{2}(u)=g_{2}(u)=u$. Rewrite Eq. (2.1) as the following system:

$$
\frac{d}{d t}[X(t)-C X(t-\tau)]=A X(t)+B_{1} f(X(t-\tau))+B_{2} g(X(t-\tau)),
$$

with

$$
\begin{array}{ll}
A=\left(\begin{array}{ccc}
-1 & 0 & 0 \\
0 & -1 & 0 \\
0 & 0 & -1
\end{array}\right), & B_{1}=\left(\begin{array}{lll}
a & 0 & 0 \\
0 & a & 0 \\
0 & 0 & a
\end{array}\right), \\
B_{2}=\left(\begin{array}{lll}
0 & b & b \\
0 & 0 & b \\
0 & b & 0
\end{array}\right), & C=\left(\begin{array}{ccc}
a_{2} & b_{2} & b_{2} \\
0 & a_{2} & b_{2} \\
0 & b_{2} & a_{2}
\end{array}\right) .
\end{array}
$$

Let $Y(t)=X(\tau t)$, then Eq. (3.1) becomes

$$
\frac{d}{d t}[Y(t)-C Y(t-1)]=A \tau Y(t)+B_{1} \tau f(Y(t-1))+B_{2} \tau g(Y(t-1)) .
$$

The characteristic equation associated with the linearization of system (3.2) around the origin is given by

$$
(\gamma E-A \tau) e^{\gamma}-C \gamma-\left(B_{1}+B_{2}\right) \tau=0 .
$$

Comparing with the previous characteristic equation, we find $\gamma=\lambda \tau$. For convenience, we denote $\gamma=\left(\tau_{j}+v\right) \lambda$, where $\tau_{j}=\tau_{s j}(s=1,2,3 ; j=0,1,2, \ldots)$ and $v \in R$. According to Theorem 1, we know that system (3.2) undergoes a Hopf bifurcation at the origin when $v=0$.

For $\varphi \in C\left([-1,0], R^{3}\right)$, let $D(\phi)=\phi(0)-C \phi(-1), L(\nu, \varphi)=A\left(\tau_{j}+\nu\right) \phi(0)+\left(B_{1}+B_{2}\right)\left(\tau_{j}+\right.$ v) $\phi(-1)$ and

$$
F(v, \phi)=\left(\tau_{j}+v\right)\left(\frac{f^{\prime \prime \prime}(0)}{6} B_{1}+\frac{g^{\prime \prime \prime}(0)}{6} B_{2}\right) \phi^{3}(-1)+\cdots .
$$

By the Riesz representation theorem, there exist functions $\eta(\theta)$ and $\mu(\theta)$ such that

$$
D(\tau, \phi)=\phi(0)-\int_{-\tau}^{0} d \mu(\theta) \phi(\theta), \quad L(\tau, \phi)=\int_{-\tau}^{0} d \eta(\theta) \phi(\theta) .
$$

In fact, we can choose

$$
\mu(\theta)=\left\{\begin{array}{ll}
-C, & \theta=0, \\
0, & \theta \in(-1,0]
\end{array} \quad \text { and } \quad \eta(\theta)= \begin{cases}-\left(B_{1}+B_{2}\right)\left(\tau_{j}+v\right), & \theta=-1, \\
0, & \theta \in(-1,0), \\
A\left(\tau_{j}+v\right), & \theta=0 .\end{cases}\right.
$$


Define

$$
A(\tau) \phi= \begin{cases}\frac{d \phi(\theta)}{d \theta}, & \theta \in[-1,0), \\ \phi^{\prime}(\theta)-C \phi^{\prime}(\theta)+L \phi, & \theta=0\end{cases}
$$

and

$$
R(\tau) \phi= \begin{cases}0, & \theta \in[-1,0), \\ F(\nu, \phi), & \theta=0 .\end{cases}
$$

Then Eq. (3.2) can be written as the abstract ODE, i.e.,

$$
\dot{Y}_{t}=A(\tau) Y_{t}+R(\tau) Y_{t}
$$

The adjoint operator $A^{*}$ is defined by $A^{*}=-\frac{d \psi}{d s}$ with the domain

$$
D\left(A^{*}\right)=\left\{\psi \in C^{*}=C\left([0, \tau], R^{3}\right): D \frac{d \psi}{d s} \in C^{*} ; D \frac{d \psi}{d s}=-L \psi\right\}
$$

We define the bilinear form:

$$
\begin{aligned}
\langle\psi, \phi\rangle= & \bar{\psi}(0) \phi(0)-\int_{-1}^{0} d\left[\int_{a=0}^{\theta} \bar{\psi}(\theta-a) d \mu(a)\right] \phi(\theta) \\
& -\int_{-1}^{0} \int_{\theta=0}^{s} \bar{\psi}(\theta-s) d \eta(a) \phi(\theta) d \theta .
\end{aligned}
$$

It is not difficult to verify that $q(\theta)=q_{0} e^{i \omega_{0} \tau_{j} \theta}(\theta \in[-1,0])$ and $q^{*}(s)=l q_{0}^{*} e^{i \omega_{0} \tau_{j} s}(s \in[0,1])$ are the eigenvectors of $A(0)$ and $A^{*}$ corresponding to the eigenvalues $i \omega_{0} \tau_{j}$ and $-i \omega_{0} \tau_{j}$, respectively, where

$$
l=\left[q_{0}^{*}\left(I-C e^{-i \omega_{0} \tau_{j}}+\left(B_{1}+B_{2}\right) \tau_{j} e^{-i \omega_{0} \tau_{j}}\right) \bar{q}_{0}\right]^{-1},
$$

and $\left\langle q^{*}, q\right\rangle=1$. Now we compute the center manifold $C_{0}$ at $v=0$. Define

$$
z(t)=\left(q^{*}, y_{t}\right), \quad W(t, \theta)=y_{t}(\theta)-2 \operatorname{Re}\{z(t) q(\theta)\}
$$

then we have

$$
\dot{z}(t)=i \omega_{0} \tau_{j} z+\bar{q}^{*}(0) F\left(0, y_{t}\right)
$$

Equation (3.2) can be written in the abbreviated form

$$
\dot{z}(t)=i \omega_{0} \tau_{j} z+g(z, \bar{z})
$$

with

$$
g(z, \bar{z})=g_{20} \frac{z^{2}}{2}+g_{11} z \bar{z}+g_{02} \frac{\bar{z}^{2}}{2}+g_{21} \frac{z^{2} \bar{z}}{2}+\cdots .
$$


Noting that $y_{t}(\theta)=W(t, \theta)+z(t) q(\theta)+\bar{z}(t) \bar{q}(\theta)$, we have

$$
\begin{aligned}
y_{t}(-1) & =\left(\begin{array}{l}
q_{01} e^{-i \omega_{0} \tau_{j}} z+\bar{q}_{01} e^{i \omega_{0} \tau_{j}} \bar{z}+W_{20}^{(1)}(-1) \frac{z^{2}}{2}+W_{11}^{(1)}(-1) z \bar{z}+W_{02}^{(1)}(-1) \frac{\bar{z}^{2}}{2}+\cdots \\
q_{02} e^{-i \omega_{0} \tau_{j}} z+\bar{q}_{02} e^{i \omega_{0} \tau_{j}} \bar{z}+W_{20}^{(2)}(-1) \frac{z^{2}}{2}+W_{11}^{(2)}(-1) z \bar{z}+W_{02}^{(2)}(-1) \frac{\bar{z}^{2}}{2}+\cdots \\
q_{03} e^{-i \omega_{0} \tau_{j}} z+\bar{q}_{03} e^{i \omega_{0} \tau_{j}} \bar{z}+W_{20}^{(3)}(-1) \frac{z^{2}}{2}+W_{11}^{(3)}(-1) z \bar{z}+W_{02}^{(3)}(-1) \frac{\bar{z}^{2}}{2}+\cdots
\end{array}\right) \\
& \stackrel{\operatorname{def}}{=}\left(\begin{array}{l}
y_{1}(-1) \\
y_{2}(-1) \\
y_{3}(-1)
\end{array}\right)
\end{aligned}
$$

where $q_{0}=\left(q_{01}, q_{02}, q_{03}\right)^{T}$. Therefore, from (3.4), we have

$$
\begin{aligned}
F\left(0, y_{t}\right) & =\tau_{j}\left(\frac{f^{\prime \prime \prime}(0)}{6} B_{1}+\frac{g^{\prime \prime \prime}(0)}{6} B_{2}\right) y_{t}^{3}(-1)+\cdots \\
& \stackrel{\text { def }}{=} F_{z^{2}} \frac{z^{2}}{2}+F_{\bar{z}^{2}} \frac{\bar{z}^{2}}{2}+F_{z \bar{z}} z \bar{z}+F_{z^{2}} \frac{z^{2} \bar{z}}{2}+\cdots
\end{aligned}
$$

with $y_{t}^{3}(-1)=\left(y_{1}^{3}(-1), y_{2}^{3}(-1), y_{3}^{3}(-1)\right)^{T}$. Substituting the expression of $F\left(0, y_{t}\right)$ into Eq. (3.6) and comparing its coefficients with that of Eq. (3.7) gives that

$$
g_{20}=g_{02}=g_{11}=0
$$

and

$$
g_{21}=\bar{q}^{*}(0) F_{z^{2} \bar{z}}=\bar{l} \bar{q}_{0}^{*} \tau_{j}\left(\frac{f^{\prime \prime \prime}(0)}{6} B_{1}+\frac{g^{\prime \prime \prime}(0)}{6} B_{2}\right)\left(\begin{array}{l}
q_{01}^{2} \bar{q}_{01} \\
q_{02}^{2} \bar{q}_{02} \\
q_{03}^{2} \bar{q}_{03}
\end{array}\right) e^{-i \omega_{0} \tau_{j}}
$$

It is well known that the coefficient $c_{1}(0)$ of third degree term of Poincaré normal form of Eq. (3.6) is given by [4]

$$
c_{1}(0)=\frac{i}{2 \omega_{0} \tau_{j}}\left(g_{20} g_{11}-2\left\|g_{11}\right\|^{2}-\frac{1}{3}\left\|g_{02}\right\|^{2}\right)+\frac{g_{21}}{2} .
$$

Consequently, we have the following theorem on the bifurcating periodic solution.

Theorem 2 For system (3.1), assume $0<a_{2}<\frac{\sqrt{2}}{2}, a<-\frac{1}{a_{2}}$, then

(1) if $f^{\prime \prime \prime}(0)>0$, the direction of the Hopf bifurcation at $\tau=\tau_{1 j}$ is supercritical and the bifurcating periodic solutions are asymptotically stable;

(2) if $f^{\prime \prime \prime}(0)<0$, the direction of the Hopf bifurcation at $\tau=\tau_{1 j}$ is subcritical and the bifurcating periodic solutions are unstable.

Proof When $\tau=\tau_{1 j}$, by calculation, we easily obtain the following results:

$$
c_{1}(0)=-\frac{a f^{\prime \prime \prime}(0) \tau_{j}}{12} \frac{1}{\left\|e^{-i \omega_{0} \tau_{j}}\left(a_{2}-a \tau_{j}\right)-1\right\|^{2}}\left[e^{-i \omega_{0} \tau_{j}}\left(a_{2}-a \tau_{j}\right)-1\right] e^{-i \omega_{0} \tau_{j}}
$$

and

$$
\operatorname{Re} c_{1}(0)=\frac{a f^{\prime \prime \prime}(0) \tau_{j}}{12} \frac{1}{\left\|e^{-i \omega_{0} \tau_{j}}\left(a_{2}-a \tau_{j}\right)-1\right\|^{2}}\left[\cos \omega_{0} \tau_{j}-\left(a_{2}-a \tau_{j}\right) \cos 2 \omega_{0} \tau_{j}\right]
$$


If $0<a_{2}<\frac{\sqrt{2}}{2}, a<-\frac{1}{a_{2}}$ and $f^{\prime \prime \prime}(0)>0(<0)$, then $\operatorname{Re} c_{1}(0)<0(>0)$. Therefore, from $\alpha^{\prime}\left(\tau_{1 j}\right)>$ 0 , we have

$$
\mu_{2}=-\frac{\operatorname{Re}\left\{c_{1}(0)\right\}}{\alpha^{\prime}\left(\tau_{1 j}\right)}>0(<0), \quad \beta_{2}=2 \operatorname{Re}\left\{c_{1}(0)\right\}<0(>0) .
$$

This completes the proof of Theorem 2 .

Similarly, we can prove Theorem 3 and Theorem 4, we omit the proof here.

Theorem 3 For system (3.1), assume $0<a_{2}+b_{2}<\frac{\sqrt{2}}{2}, a+b<-\frac{1}{a_{2}+b_{2}}$, then

(1) if $a f^{\prime \prime \prime}(0)+b g^{\prime \prime \prime}(0)>0$, the direction of the Hopf bifurcation at $\tau=\tau_{2 j}$ is subcritical and the bifurcating periodic solutions are unstable;

(2) if af $f^{\prime \prime \prime}(0)+b g^{\prime \prime \prime}(0)<0$, the direction of the Hopf bifurcation at $\tau=\tau_{2 j}$ is supercritical and the bifurcating periodic solutions are asymptotically stable.

Theorem 4 For system (3.1), assume $0<a_{2}-b_{2}<\frac{\sqrt{2}}{2}, a-b<-\frac{1}{a_{2}-b_{2}}$, then

(1) if $a f^{\prime \prime \prime}(0)-b g^{\prime \prime \prime}(0)>0$, the direction of the Hopf bifurcation at $\tau=\tau_{3 j}$ is subcritical and the bifurcating periodic solutions are unstable;

(2) if $a f^{\prime \prime \prime}(0)-b g^{\prime \prime \prime}(0)<0$, the direction of the Hopf bifurcation at $\tau=\tau_{3 j}$ is supercritical and the bifurcating periodic solutions are asymptotically stable.

\section{Permanent oscillation}

Based on Chafee's criterion, if system (2.1) has a unique equilibrium point which is unstable, and the solutions of system (2.1) are globally bounded, this system generates a limit cycle, namely a permanent oscillation [5].

We consider system (2.1) and assume that $f, g, f_{2}, g_{2}$ are nonlinear bounded functions and satisfy Lipschitz condition,

$$
\frac{|f(x)-f(y)|}{|x-y|} \leq L_{1}, \quad \frac{|g(x)-g(y)|}{|x-y|} \leq L_{2} .
$$

We have the following lemmas.

Lemma 5 If $|\alpha| L_{1}+|\beta| L_{2}<1$ holds, system (2.1) has a unique equilibrium point.

Proof Suppose that $X^{*}$ is the equilibrium point of the system, then we have

$$
A X^{*}+B_{1} f\left(X^{*}\right)+B_{2} g\left(X^{*}\right)=0 \text {. }
$$

We define a mapping $H: R^{3} \rightarrow R^{3}$

$$
H(X)=A X+B_{1} f(X)+B_{2} g(X)
$$

and assume $H(u)=H(v)$, then

$$
\Phi(u-v)=\left(\begin{array}{ccc}
-1+a c_{1} & d_{2} b & d_{3} b \\
0 & -1+a c_{2} & d_{3} b \\
0 & d_{2} b & -1+a c_{3}
\end{array}\right)(u-v)=0
$$


where $\left|c_{i}\right| \leq L_{1},\left|d_{j}\right| \leq L_{2}(i=1,2,3 ; j=2,3)$. Under the given condition, $\Phi$ is an invertible matrix. Then $u=v$, namely $H(X)$ is injective on $R^{3}$. Noting that $f$ and $g$ are bounded continuous functions, it is easily to obtain that $\|H(u)\| \rightarrow \infty$, when $\|u\| \rightarrow \infty$. So $H(X)$ is a homeomorphism on $R^{3}$ and system (2.1) has a unique equilibrium point.

Lemma 6 The solutions of system (2.1) are globally bounded.

Proof Since $f, g, f_{2}$ and $g_{2}$ are bounded continuous functions, there is $M>0$ such that

$$
\frac{d\left|x_{i}(t)\right|}{d t} \leq-\left|x_{i}(t)\right|+M
$$

with $i=1,2,3$. This proves the lemma.

Lemma 7 The equilibrium point (0,0,0) of system (2.1) is unstable when one of the following conditions are satisfied:

(1) $\alpha_{2}>0, \alpha_{2}>\alpha \tau$, and $\tau+\left(1-\frac{\alpha}{\alpha_{2}} \tau\right)<\alpha_{2} e^{-\left(1-\frac{\alpha}{\alpha_{2}} \tau\right)}$,

(2) $\alpha_{2}+\beta_{2}>0, \alpha_{2}+\beta_{2}>(\alpha+\beta) \tau$, and $\tau+\left(1-\frac{\alpha+\beta}{\alpha_{2}+\beta_{2}} \tau\right)<\left(\alpha_{2}+\beta_{2}\right) e^{-\left(1-\frac{\alpha+\beta}{\alpha_{2}+\beta_{2}} \tau\right)}$,

(3) $\alpha_{2}-\beta_{2}>0, \alpha_{2}-\beta_{2}>(\alpha-\beta) \tau$, and $\tau+\left(1-\frac{\alpha-\beta}{\alpha_{2}-\beta_{2}} \tau\right)<\left(\alpha_{2}-\beta_{2}\right) e^{-\left(1-\frac{\alpha-\beta}{\alpha_{2}-\beta_{2}} \tau\right)}$.

Proof Based on analysis in [2], we know the roots of the following equation:

$$
\lambda+1-\left(\alpha+\alpha_{2} \lambda\right) e^{-\lambda \tau}=0
$$

are the characteristic roots of the linearized system of (2.1). When condition (1) holds, Eq. (4.1) has at least a positive real root, and the equilibrium point $(0,0,0)$ of system $(2.1)$ is unstable.

Using the same method, we can obtain conditions (2) and (3). The proof of the lemma is completed.

Up to now, we have prepared sufficiently to state the following results.

Theorem 8 System (2.1) generates a permanent oscillation, when $|\alpha| L_{1}+|\beta| L_{2}<1$ holds and one of the following conditions are satisfied:

(1) $\alpha_{2}>0, \alpha_{2}>\alpha \tau$, and $\tau+\left(1-\frac{\alpha}{\alpha_{2}} \tau\right)<\alpha_{2} e^{-\left(1-\frac{\alpha}{\alpha_{2}} \tau\right)}$,

(2) $\alpha_{2}+\beta_{2}>0, \alpha_{2}+\beta_{2}>(\alpha+\beta) \tau$, and $\tau+\left(1-\frac{\alpha+\beta}{\alpha_{2}+\beta_{2}} \tau\right)<\left(\alpha_{2}+\beta_{2}\right) e^{-\left(1-\frac{\alpha+\beta}{\alpha_{2}+\beta_{2}} \tau\right)}$,

(3) $\alpha_{2}-\beta_{2}>0, \alpha_{2}-\beta_{2}>(\alpha-\beta) \tau$, and $\tau+\left(1-\frac{\alpha-\beta}{\alpha_{2}-\beta_{2}} \tau\right)<\left(\alpha_{2}-\beta_{2}\right) e^{-\left(1-\frac{\alpha-\beta}{\alpha_{2}-\beta_{2}} \tau\right)}$.

\section{Numerical simulation}

In the section, we carry out some numerical simulations for system (2.1).

Assume that $\alpha=0.1, \beta=0.4, \alpha_{2}=0.2$ and $\beta_{2}=0.2$. From Theorem 1 , the zero solution of system (2.1) is absolutely stable. The simulation results as shown in Figure 2.

Assume that $\alpha=-0.8, \beta=-1.3, \alpha_{2}=0.2$, and $\beta_{2}=0.5$. From Theorem 1 , the zero solution of system (2.1) is conditionally stable, i.e., $\tau \in\left[0, \tau_{20}=0.48\right)$, the zero solution of system (2.1) is asymptotically stable; $\tau>\tau_{20}=0.48$, the zero solution of system (2.1) is 

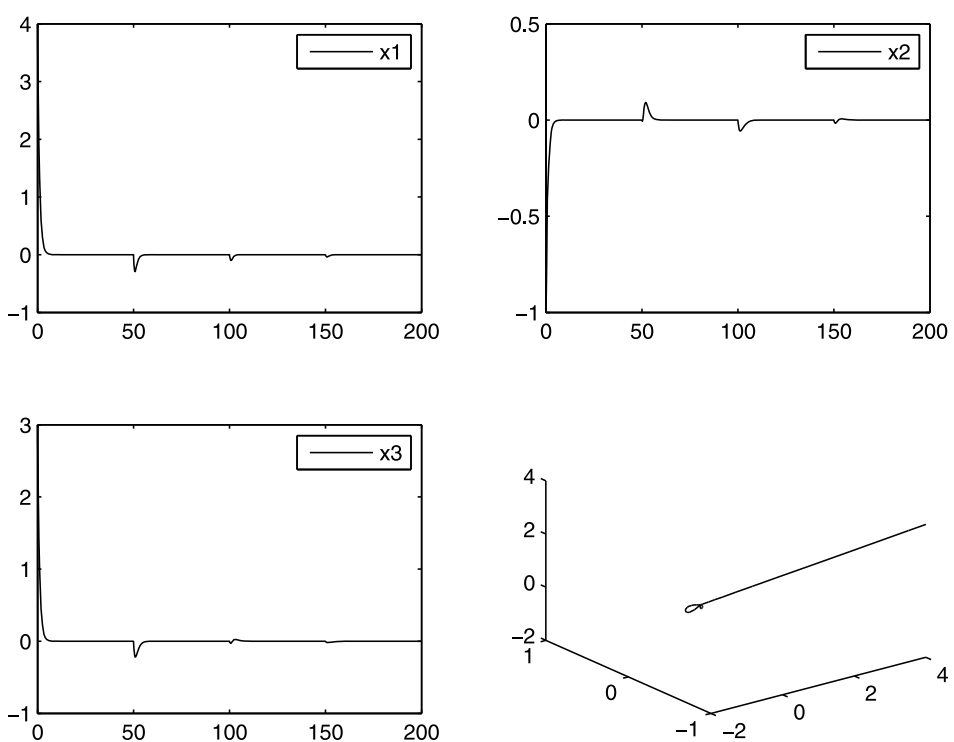

Figure 2 For system (2.1), when $\tau=50$, the zero solution is asymptotically stable.
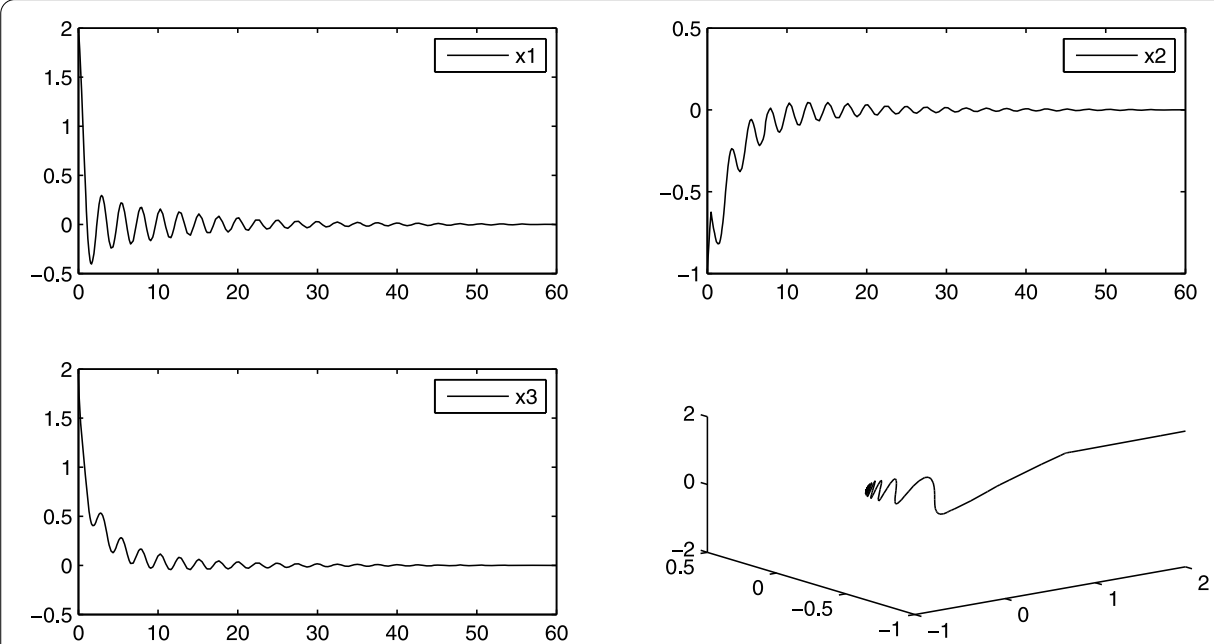

Figure 3 For system (2.1), when $\tau=0.47<\tau_{20}=0.48$, the zero solution is asymptotically stable.

unstable, and system (2.1) undergoes a Hopf bifurcation at the origin when $\tau=\tau_{20}$. Furthermore, the direction of the Hopf bifurcation at $\tau=\tau_{2 j}$ is subcritical and the bifurcating periodic solutions are unstable. The simulation results as shown in Figures 3 and 4.

Consider system (2.1) with $\alpha=0.2, \beta=0.6, \alpha_{2}=3$ and $\beta_{2}=2$, then we can choose $\tau=1$, satisfying Theorem 8 . System (2.1) generates a permanent oscillation (see Figure 5 ).

\section{Conclusion}

For a neutral model including three cells with time delay, we have given the general condition for the stability and shown the delay-independent and delay-dependent local stability regions. We have also obtained the condition to determine the direction of Hopf bifurcations, the stability of bifurcating periodic solutions and a permanent oscillation. 

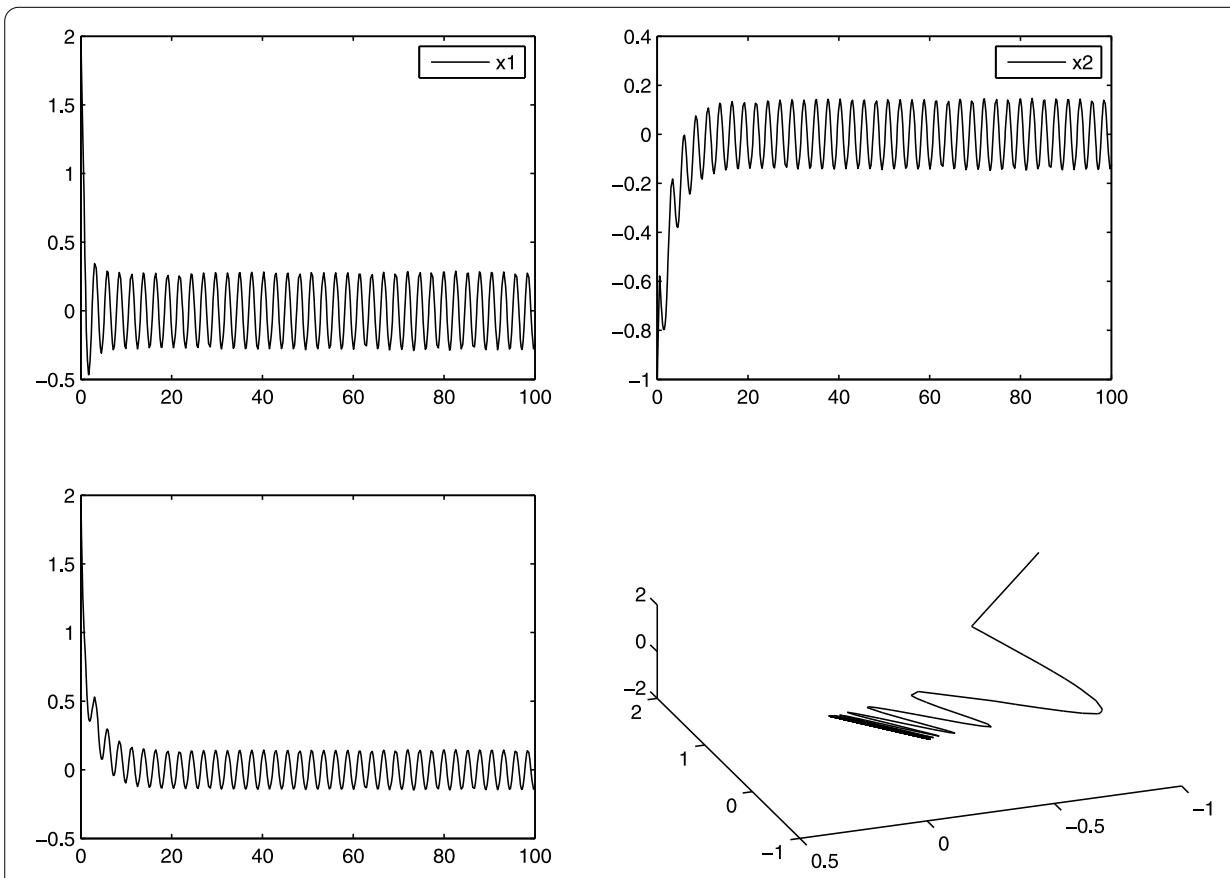

Figure 4 For system (2.1), the bifurcating periodic solution is unstable when $\tau=0.54>\tau_{20}=0.48$.
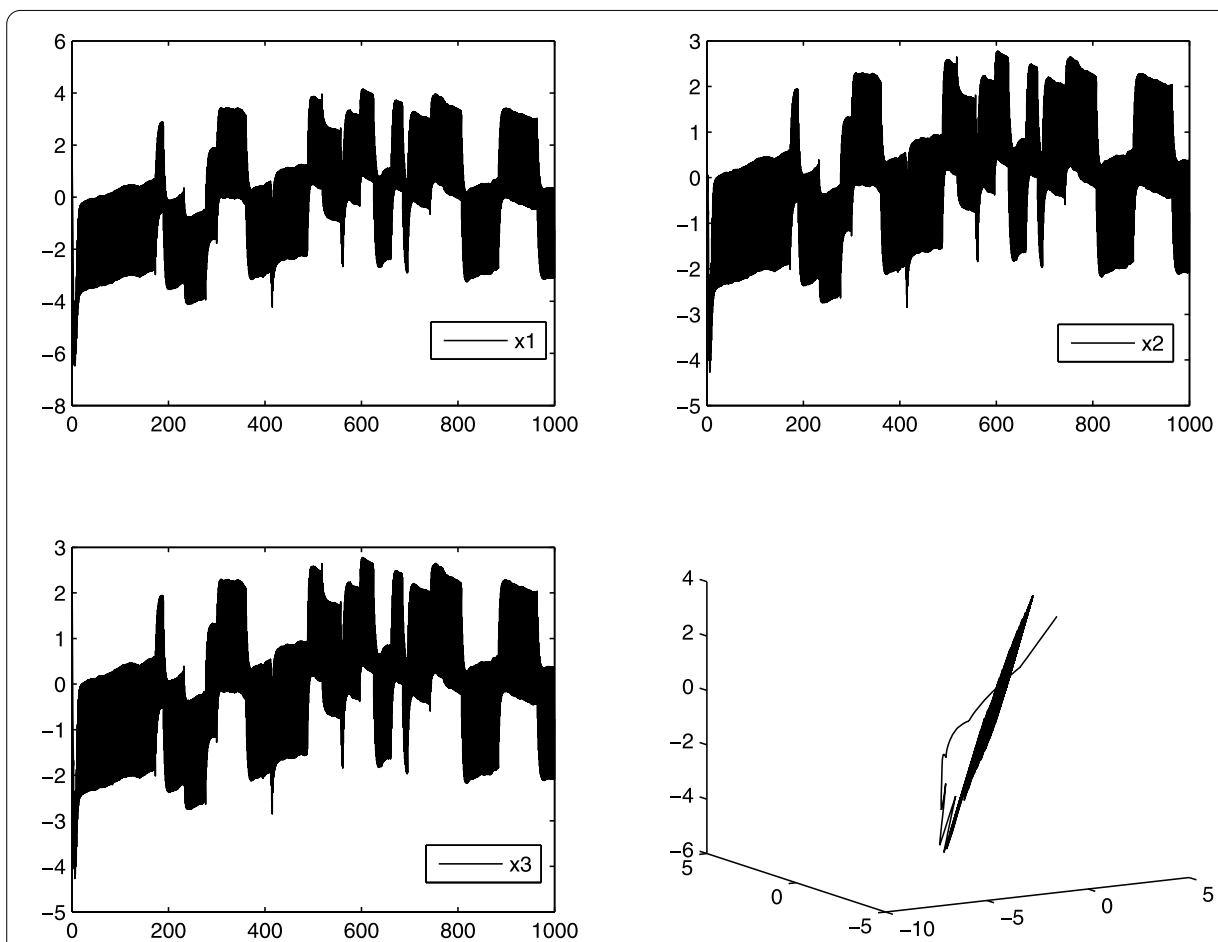

Figure 5 System (2.1) generates a permanent oscillation. 
As we know, the extension of local periodic solutions for large time delay would appear when some conditions are satisfied. Further study of the patterns is undergoing.

\section{Competing interests}

The authors declare that they have no competing interests.

\section{Authors' contributions}

The authors have achieved equal contributions to each part of this paper. All the authors read and approved the final manuscript.

\section{Acknowledgements}

The authors are very grateful to the referees for their valuable suggestions. This work is supported by the Fundamental Research Funds for the Central Universities (No. DL12BB29) and the National Natural Science Foundation of China (Grant No. 41304093).

Received: 31 January 2013 Accepted: 28 October 2013 Published: 22 Nov 2013

\section{References}

1. Li, L, Yuan, Y: Dynamics in three cells with multiple time delays. Nonlinear Anal., Real World Appl. 9, $725-746$ (2008)

2. Luo, S, Liu, M, Xu, X: Stability analysis of class of neutral three neurons network with delay. J. Nat. Sci. Heilongjiang Univ. 3, 336-340 (2010)

3. Wei, J, Ruan, S: Stability and global Hopf bifurcation for neutral differential equations. Acta Math. Sin. 45, 94-104 (2002)

4. Hassard, B, Kazarinoff, N, Wan, Y: Theory and Applications of Hopf Bifurcation. Cambridge University Press, Cambridge (1981)

5. Chafee, N: A bifurcation problem for a functional differential equation of finitely retarded type. J. Math. Anal. Appl. 35, 312-348 (1971)

10.1186/1687-1847-2013-338

Cite this article as: Liu et al.: Dynamics of a class of neutral three neurons network with delay. Advances in Difference Equations 2013, 2013:338

\section{Submit your manuscript to a SpringerOpen ${ }^{\circ}$ journal and benefit from:}

- Convenient online submission

- Rigorous peer review

Immediate publication on acceptance

Open access: articles freely available online

- High visibility within the field

- Retaining the copyright to your article 